\title{
Modeling of Human Driving Behavior Based on Expression as Hybrid Dynamical System ${ }^{\dagger}$
}

\author{
Jong-Hae KIm*, Soichiro HaYaKaWA**, Tatsuya Suzuki*, \\ Kazuaki HiranA $^{* * *}$, Yoshimichi Matsui*, Shigeru OKumA*, \\ Nuio TsuchidA**, Masayuki ShImIzU**** and Shigeyuki KIDO****
}

This paper develops a new framework to understand the human driving behavior based on the expression as a Hybrid Dynamical System(HDS) focusing on the driver's stopping maneuver. The driving data are collected by using the three-dimensional driving simulator based on CAVE, which provides three three-dimensional visual information. In our modeling, the relationship between the measured information such as distance to the stop line, its first and second derivatives and the braking amount are expressed by the Piece-Wise Polynomial System (PWPS) model, which is a class of HDS. The key idea to solve the identification problem is to formulate the problem as the Mixed Integer Linear Programming (MILP) with replacing the switching conditions by binary variables. From the obtained results, it is found that the driver appropriately switches the 'control law' according to the distance to the stop line. Our proposed approach enables us to capture not only the physical meaning of the driving skill, but also the decision-making aspect (switching conditions) in the driving behavior.

Key Words: CAVE, virtual reality, three-dimensional visual information, PWPS, MILP, driving skill

\section{Introduction}

Recently, the modeling of the driving behavior has attracted a great deal of attention by many researchers 1) 3). Since the driving behavior usually includes high non-linearity, some nonlinear dynamics based modeling ${ }^{6)}$ is promising. From this viewpoint, the nonlinear regression models, the neural network and the fuzzy system etc. can be used. If we use these techniques, however, the obtained model often results in too complicated model, and this makes it impossible to understand the physical meaning of the driver's behavior. When we look at the driving behavior, it is often found that the driver appropriately switches the simple control laws instead of adopting complex nonlinear control law. This switching mechanism can be regarded as a kind of driver's decision making in the driver's behavior.

$\dagger$ The paper was presented at the ICCAS conference(October 2003)

* Nagoya University, Furo-cho, Chikusa-ku, Nagoya

** Toyota Technological Institute, Hisakata, Tempaku-ku, Nagoya

*** Chukyo University, Tokodachi, Kaizu-cho, Toyota

**** Toyota Motor Corporation, Toyota-cho 1, Toyota (Received June 30, 2003)

(Revised November 7, 2003)
Therefore, it is highly recommended that the model of the driving behavior involve both physical skill (motion) and the decision-making aspect (switching condition). This kind of expression can be categorized into a class of Hybrid Dynamical Systems (HDSs). HDSs are systems, which consist of both continuous dynamics and logical conditions. The former are typically associated with the differential (or difference) equations, the latter with combinatorial logics, automata and so on. Although many literatures have dealt with the description, stability analysis, control and verification of the HDS, the identification problem for the HDS, that is finding the HDS model to represent the measured data, has not been fully discussed in the community of the HDS ${ }^{4) \sim 7)}$.

In this paper, the Piece-Wise Polynomial Systems (PWPS) model, which is a class of the HDS, is adopted to understand the human driving behavior especially focusing on the stopping maneuver. The driving data are collected by using the three-dimensional driving simulator ${ }^{2)}{ }^{13)}$ based on CAVE, which provides three-dimensional visual information. The major difference between our simulator and the real environment is the existence of the real motion of the vehicle. This implies that the acceleration factor may affect on the driving maneuver in different way. However, thanks to the 3D effect of the CAVE, this dif- 
ferece is not so large, i.e. the examinee shows the similar behavior in the stopping maneuver with one in the real environment ${ }^{2)}$.

In our modeling, the relationship between the measured information such as distance to the stop line, its first and second derivatives and the braking amount are expressed by the PWPS model. Then, we formulate the identification problem for the PWPS model as the Mixed Integer Linear Programming (MILP) by transforming the logical conditions into inequalities. By applying the developed strategy, it becomes possible to find not only coefficients in the polynomials but also parameters in the logical conditions from the measured driving data. This implies that both physical skill and the dccision-making aspect in the driving bchavior can bc identified simultaneously.

This paper is organized as follows.

In Section 2, configuration of the developed DS based on the CAVE is introduced. In Section 3, the scenario of our examination is described. Based on the setup described in Section 3, driver's stopping maneuver is investigated in Section 4. In Section 5, the modeling of the stopping maneuver based on the expression as the PWPS model is
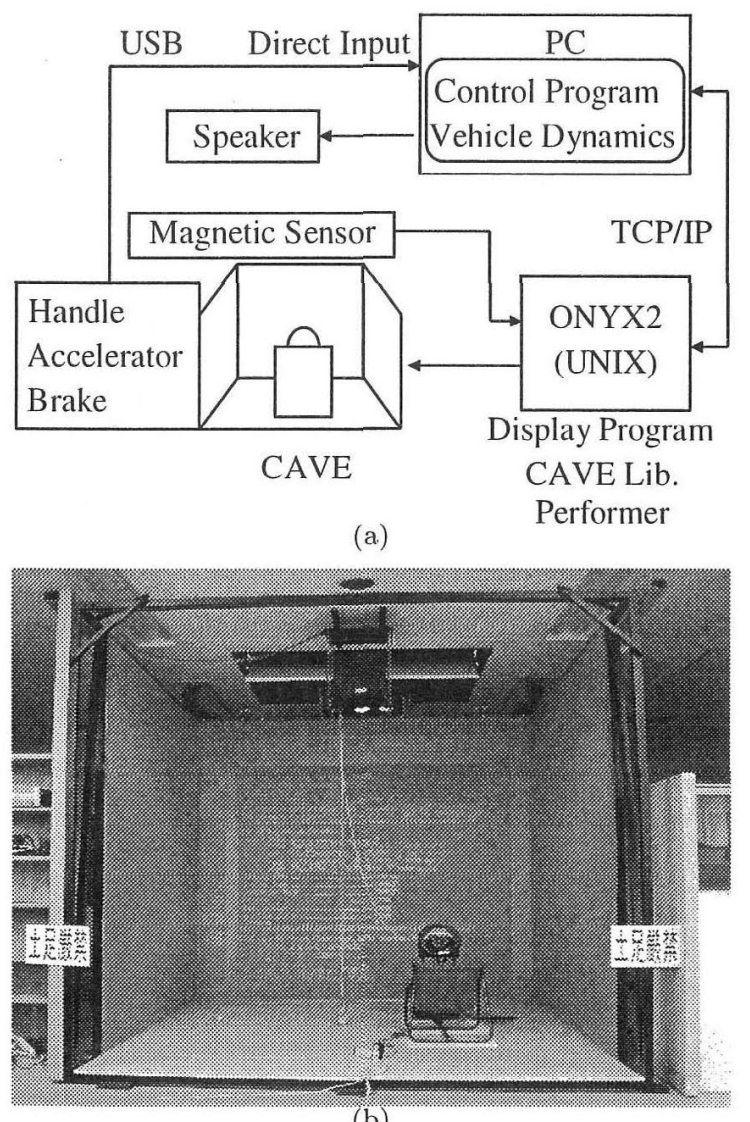

(b)

Fig. 1 The developed driving simulator. (a) Configuration of the DS.(b) CAVE system. introduced, and identification results based on the MILP are shown.

\section{Configuration of Driving Simulator}

The configuration and appearance of the developed DS based on the CAVE are shown in Fig. 1(a) and (b). The display unit in the CAVE system provides the $3 \mathrm{D}$ virtual environment, and it is controlled by ONYX2.

The display program was developed by making use of the CAVE library and the Performer. The cockpit is built by installing a handle, an accelerator and a brake in the CAVE system.

The information on the driver's output to the handle, accelerator and brake is transferred to the PC through the USB tcrminal, and the vehicle position and motion are calculated based on these inputs and vehicle dynamics implemented on the PC using the CarSim software. The results of the calculation are transferred to ONYX2 through the Internet (TCP/IP), and the 3D visual image based on the position and motion of the vehicle is displayed.

\section{Capturing Driving Behavior}

\subsection{Road environment and investigated task}

Generally speaking, most traffic accidents occur at intersections ${ }^{8), 9)}$. In this paper, we focus on a stopping maneuver in front of the stop line because this maneuver strongly depends on the distance to the stop line, and our DS has clear advantage over other $2 \mathrm{D}$ virtual environment based $\mathrm{DS}^{2)}$.

In order to model the stopping maneuver, the following sensory information is captured as the inputs:

(i) Distance from the vehicle to the stop line $\left[x_{1, k}\right]$

(ii) First time derivative of (i) (velocity) $\left[x_{2, k}\right]$

(iii) Second time derivative of (i) (acceleration) $\left[x_{3, k}\right]$

The outputs of the driver are also specified as follows:

(i) Acceleration $\left[y_{1, k}\right]$

(ii) Braking $\left[y_{2}, k\right]$

Note that no steering operation is necessary in the stopping maneuver.

Since it is reported that the velocity $x_{2, k}$ does not play an important role in the stopping maneuver ${ }^{2), 10), 11)}$, (i) and (iii) of the sensory information and (ii) of the output of the driver are used for the modeling.

The configuration of the intersection and its projected image are shown in Fig. 2(a) and (b) with some geometric parameters.

The road is $7 \mathrm{~m}$ wide and the pedestrian way is $2 \mathrm{~m}$ wide. There are two intersections in this environment ( $\mathrm{A}$ and 
B). The driver is supposed to stop in front of the stop line at the intersection (A). The vehicle is supposed to start moving at position (a or b).

\section{2 Driving conditions}

The vehicle used in the simulator is a large-size passenger car whose engine displacement is $3000 \mathrm{cc}$. Eight male drivers who have driver's licenses, ranging in age from 22 to 25 years, are selected as examinees. All examinees did not know the purpose of this experiment and we did not allow them to have any knowledge regarding this study. The maximum velocity is set to be $50 \mathrm{~km} / \mathrm{h}$, and the selected starting point is $0 \mathrm{~m}$ or $100 \mathrm{~m}$ as shown in Fig. 2(a).

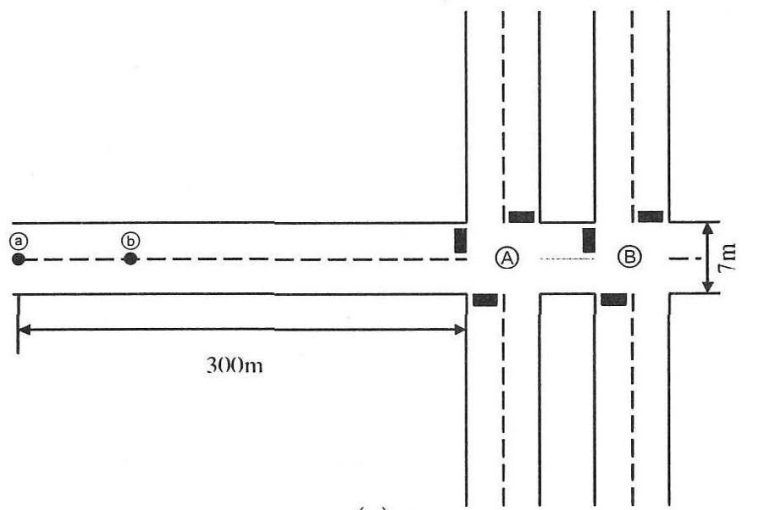

(a)

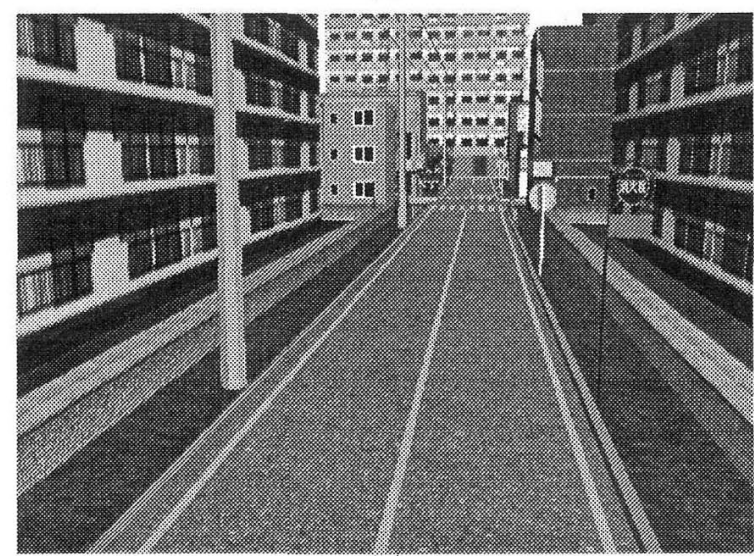

(b)

Fig. 2 Approaching an intersection.(a) Model of intersection.(b) Sample of projected image.

Table 1 Experimental Conditions

\begin{tabular}{c|c|c}
\hline \hline \multirow{2}{*}{ Notation } & \multicolumn{2}{|c}{$\begin{array}{c}\text { Driving conditions(Maximum steady } \\
\text { running velocity }[\mathrm{km} / \mathrm{h}], \text { start point }[\mathrm{m}] \text { ) }\end{array}$} \\
\cline { 2 - 3 } & $50 \mathrm{~km} / \mathrm{h} 0 \mathrm{~m}$ & $50 \mathrm{~km} / \mathrm{h} 100 \mathrm{~m}$ \\
\hline Preliminary drive & Ten times & Ten times \\
\hline Test drive & Three times & Three times \\
\hline \hline
\end{tabular}

Each driver takes a number of preliminary trials to get used to the DS, and then begins the test, which is made up of numerous trials. The number of trials for each driver is listed in Table $\mathbf{1}$.

\subsection{Experimental procedures}

Both preliminary trials and test trials are carried out twenty times and six times, respectively. These trials consist of two different driving situations.

(1) Velocity restriction of $50 \mathrm{~km} / \mathrm{h}$, starting point of $0 \mathrm{~m}$.

(2) Velocity restriction of $50 \mathrm{~km} / \mathrm{h}$, starting point of $100 \mathrm{~m}$.

The experiments are executed in the sequence shown in Table 1. After the preliminary trials, the driver rests for 10 minutes, and answers a questionnaire in which he record his impressions of his driving behavior, driving preferences, SSSQ ${ }^{12)}$, and so on. The SSSQ schedule is a way to find a driver who is likely to suffer from simulator sickness. If the driver wants to quit the experiment due to the simulator sickness, the experiment is suspended. Each driver takes about 30 minutes to complete all of the trials.

\section{Experimental Results and Proposed Modeling Framework}

Based on the setup described in section 3, eight drivers carried out the experiment under virtual environments.

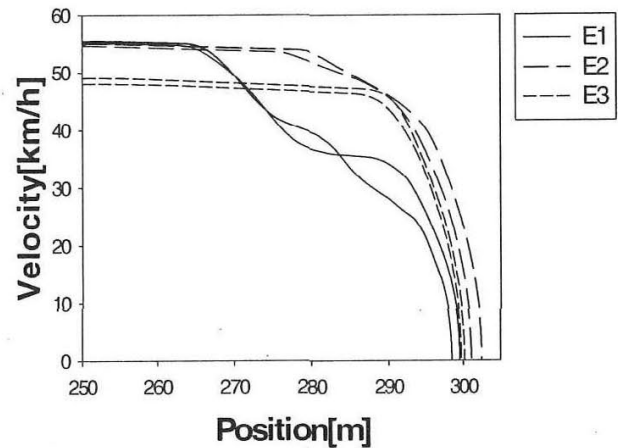

(a)

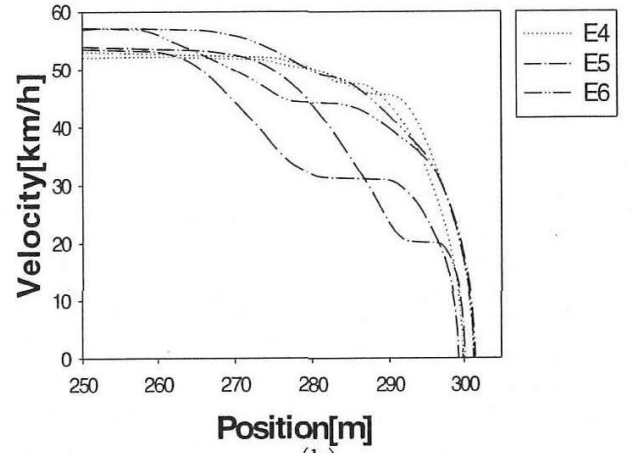

(b)

Fig. 3 Behavior at stopping

Firstly, the stopping maneuver, which is characterized by the profile between the beginning of the deceleration and the stopping point, were measured and analyzed. The profiles of the driving data of the six drivers in the case 
that the velocity restriction is $50 \mathrm{~km} / \mathrm{h}$ are depicted in Fig. 3 (the data of third and fourth trials with $50 \mathrm{~km} / \mathrm{h}$, $0 \mathrm{~m}$ and $50 \mathrm{~km} / \mathrm{h}, 100 \mathrm{~m}$, respectively).

The data of the driver who has shown high levels of simulator sickness in our questionnaires were not used for the modeling. The personal information of six examinees are listed in Table 2.

Table 2 Individual information of examinees

\begin{tabular}{c|c|c|c}
\hline Examinee & $\begin{array}{c}\text { Age } \\
\text { [Years old] }\end{array}$ & $\begin{array}{c}\text { Mileage per year } \\
{[\mathrm{km}]}\end{array}$ & $\begin{array}{c}\text { Driving Career } \\
{[\text { Years] }}\end{array}$ \\
\hline E1 & 22 & 2500 & 3 \\
\hline E2 & 22 & 15000 & 2 \\
\hline E3 & 21 & 5000 & 3 \\
\hline E4 & 22 & 5000 & 4 \\
\hline E5 & 22 & 5000 & 3 \\
\hline E6 & 22 & 25000 & 3 \\
\hline \hline
\end{tabular}

The horizontal and vertical axes in Fig. 3 denote the position (stop line is located at $300 \mathrm{~m}$ ) and the velocity of the vehicle, respectively. When we look at profiles in Fig. 3, roughly speaking, the stopping maneuver can be regarded as the series of the following three sub-maneuvers: (1) deceleration, (2) running with constant velocity and (3) re-deceleration. This sequence of sub-maneuvers is often observed in the driving pattern in real cars. In Fig. 3(a), however, the drivers E2 and E3 show different maneuver from one described above. From the answer to the questionnaire, we could find the reason of this as follows: The driver (E2) often makes 'sudden stopping' in his real driving situation. Also, the driver (E3) has found the optimal beginning point for the deceleration by investigating the building and the electric pole around the stop line instead of taking a look at the stop line in this experiment.

In the following, we call the duration corresponding to above three sub-maneuvers 'Interval A', 'Interval B' and 'Interval C'.

The more formal expression based on the polynomial model is introduced in the following.

The interval A(deceleration)

$$
\begin{gathered}
y_{2, k}=a_{0} x_{1, k}+a_{1} x_{3, k}+a_{2} x_{1, k}^{2}+a_{3} x_{3, k}^{2} \\
\text { if } d_{1}>x_{1, k}
\end{gathered}
$$

The interval B(running with constant velocity)

$$
\begin{gathered}
y_{2, k}=b_{0} x_{1, k}+b_{1} x_{3, k}+b_{2} x_{1, k}^{2}+b_{3} x_{3, k}^{2} \\
\text { if } d_{2}>x_{1, k} \geq d_{1}
\end{gathered}
$$

The interval $C$ (re-deceleration)

$$
y_{2, k}=c_{0} x_{1, k}+c_{1} x_{3, k}+c_{2} x_{1, k}^{2}+c_{3} x_{3, k}^{2}
$$

$$
\text { if } x_{1, k} \geq d_{2}
$$

In eqs. (1) to (3), $x_{1, k}$ and $x_{3, k}$ denote the position and acceleration, respectively. $d_{1}$ and $d_{2}$ are parameters to specify the switching between intervals. Note that only $x_{1, k}$ and $x_{3, k}$ appear in the left-hand side in eqs.(1) to (3). In our past study ${ }^{2)}$, we have clarified that $x_{1, k}$ and $x_{3, k}$ play important roles in the stopping maneuver by applying the GMDH technique. Also, the number of switching may be reduced to explain the behavior found in examinees E2 and E3. This behavior, however, can be explained naturally by setting the distance between $d_{1}$ and $d_{2}$ to be small. Since the structure of this model contains both the continuous dynamics (polynomials) and the logical conditions (switching of polynomials), the proposed model belongs to a kind of Hybrid Dynamical Systems (HDS).

As alternative ways of modeling the driving maneuver, for example, nonlinear regression models, neural network and fuzzy system can be used. If we use these techniques, however, the obtained model often results in too complicated model, and this makes it impossible to understand the physical meaning of the driver's behavior. On the contrary, the HDS model proposed in this section enables us to capture not only the physical meaning (polynomials), but also the decision-making aspect (logical conditions) in the driving maneuver. Note that the switching conditions between each interval are not specified in advance in our model. The parameter specifying switching condition ( $d_{1}$ and $d_{2}$ ) and coefficients appearing in each polynomial ( $a_{i}$ to $c_{i}$ ) must be found simultaneously from the measured data. In the next section, the strategy to solve this simultaneous identification problem is developed.

\section{Modeling of Driver's Behavior Based on Piece-Wise Polynomial System (PWPS) Expression}

\section{1 Expression as PWPS}

PWPS is one of classes of hybrid system, in which logic, dynamics and constraints are integrated. PWPS is formulated by (4).

$$
y_{2, k}=\left\{\begin{array}{c}
a_{0} x_{1, k}+a_{1} x_{3, k}+a_{2} x_{1, k}^{2}+a_{3} x_{3, k}^{2} \\
\text { if } d_{1}>x_{1, k} \\
b_{0} x_{1, k}+b_{1} x_{3, k}+b_{2} x_{1, k}^{2}+b_{3} x_{3, k}^{2} \\
\text { if } d_{2}>x_{1, k} \geq d_{1} \\
c_{0} x_{1, k}+c_{1} x_{3, k}+c_{2} x_{1, k}^{2}+c_{3} x_{3, k}^{2} \\
\text { if } x_{1, k} \geq d_{2}
\end{array}\right.
$$

where $k \in Z$ is a discrete time. Thus, the PWPS form includes both continuous dynamics (polynomials) and logical conditions. The goal of our modeling is to find not only coefficients in the polynomials $a_{i}, b_{i}$ and $c_{i}$ but also 
parameters in the "switching conditions" $d_{i}$ from the measured driving data. Although this identification problem is not straightforward to handle, the idea developed in the Mixed Logical Dynamical Systems framework [4] makes it tractable. The key idea is to transform the logical condition into some inequalities by introducing auxiliary binary variables $\delta \in\{0,1\}$ and auxiliary continuous variables $z$, and to formulate the problem as the Mixed Integer Linear Programming.

5. 2 Useful tools for transformation from logical condition into inequalities

In this section, some useful tools to transform the logical condition into inequalities are introduced. Firstly, the logical relationship given by

$$
[f(x) \geq a] \Leftrightarrow[\delta=1]
$$

can be transformed into inequalities (6) and (7).

$$
\begin{aligned}
& -f(x)+(a-m) \delta+m \leq 0 \\
& f(x)-(M-a+\epsilon) \delta-a+\epsilon \leq 0
\end{aligned}
$$

where $M=\max _{x} f(x), m=\min _{x} f(x)$, and $\epsilon>0$ is a small tolerance. Also, in our setup, the product term of binary and continuous variables such as $\delta f(x)$ often appear. Since it is undesirable to handle this nonlinear term, we secondly introduce another auxiliary variable $z=\delta f(x)$, which satisfies the following two logical relationships.

$$
[\delta=0] \Rightarrow[z=0],[\delta=1] \Rightarrow[z=f(x)]
$$

These relationships can be transformed into the following equivalent inequalities.

$$
\begin{aligned}
& z \leq M \delta \\
& -z \leq-m \delta \\
& z \leq f(x)-m(1-\delta) \\
& -z \leq-f(x)+M(1-\delta)
\end{aligned}
$$

\subsection{Identification of PWPS model by Mixed Integer Linear Programming(MILP)}

In order to transform the three logical conditions involved in (4) into the equivalent inequalities, binary variables $\delta_{1, k}, \delta_{2, k}$ are introduced as follows:

(1) $\left[d_{1}>x_{1, k}\right] \Leftrightarrow\left[\delta_{1, k}=0, \delta_{2, k}=0\right]$

(2) $\left[d_{2}>x_{1, k} \geq d_{1}\right] \Leftrightarrow\left[\delta_{1, k}=1, \delta_{2, k}=0\right]$

(3) $\left[x_{1, k}>d_{2}\right] \Leftrightarrow\left[\delta_{1, k}=0, \delta_{2, k}=1\right]$

By applying the transformation rules and introducing the auxiliary variables, eq. (4) can be reformulated by the following linear equation.

$$
\begin{aligned}
y_{2, k}= & a_{0} x_{1, k}+a_{1} x_{3, k}+a_{2} x_{1, k}^{2}+a_{3} x_{3, k}^{2} \\
& +x_{1, k} z_{1, k}+x_{3, k} z_{2, k}+x_{1, k}^{2} z_{3, k}+x_{3, k}^{2} z_{4, k}
\end{aligned}
$$

$$
+x_{1, k} z_{5, k}+x_{3, k} z_{6, k}+x_{1, k}^{2} z_{7, k}+x_{3, k}^{2} z_{8, k}
$$

where, the auxiliary variables, $z_{i, k}$, are defined as follows.

$$
\begin{aligned}
& z_{i, k}=\delta_{1, k} f_{i, k}=\delta_{1, k}\left(b_{i-1}-a_{i-1}\right) \quad(i=1 \sim 4) \\
& z_{i, k}=\delta_{2, k} f_{i, k}=\delta_{2, k}\left(c_{i-5}-a_{i-5}\right) \quad(i=5 \sim 8) \\
& z_{i, k}=\delta_{1, k} f_{i, k}=\delta_{1, k} d_{i-8} \quad(i=9 \sim 10) \\
& z_{i, k}=\delta_{2, k} f_{i, k}=\delta_{2, k} d_{i-10} \quad(i=11 \sim 12)
\end{aligned}
$$

Also, as stated in the previous section, some linear inequalities that come up with the introduction of $\delta_{1, k}, \delta_{2, k}$ and $z_{i, k}$ must be accompanied with (13) to (17).

Now, the problem to find the parameters in the switching condition and coefficients in the polynomials is formulated as the following MILP.

known $y_{2, k}, x_{1, k}, x_{3, k}$

find $\left\{a_{0}, a_{1}, a_{2}, a_{3}, b_{0}, b_{1}, b_{2}, b_{3}\right.$, $\left.c_{0}, c_{1}, c_{2}, c_{3}, d_{1}, d_{2}, \delta_{1, k}, \delta_{2, k}\right\}$

$$
\text { which minimize } J=\sum_{k=1}^{N}\left|y_{2, k}-\hat{y}_{2, k}\right|
$$

subject to

$$
\begin{aligned}
& z_{i, k} \leq M_{i} \delta_{j, k} \\
& -z_{i, k} \leq-m_{i} \delta_{j, k} \\
& z_{i, k} \leq f_{i, k}-\left(1-\delta_{j, k}\right) m_{i} \\
& -z_{i, k} \leq-f_{i, k}+\left(1-\delta_{j, k}\right) M_{i} \\
& \delta_{1, k}=\{0,1\} \\
& \delta_{2, k}=\{0,1\} \\
& 0 \leq \delta_{1, k}+\delta_{2, k} \leq 1 \\
& \delta_{2, k} \leq \delta_{2, k+1} \\
& x_{1, k} \leq d_{1}-z_{9, k}+z_{10, k}-z_{11, k}-\epsilon+\delta_{2, k}\left(M_{d}+\epsilon\right)(27) \\
& x_{1, k} \geq\left(1-\left(\delta_{1, k}+\delta_{2, k}\right)\right) m_{d}+z_{9, k}+z_{12, k}
\end{aligned}
$$

where, $M_{i}$ and $m_{i}(i=1 \sim 12)$ are the maximum and minimum values of $z_{i, k}$, and $M_{d}$ and $m_{d}$ represent the maximum and minimum values of $d_{1}$ and $d_{2}$, respectively, $\epsilon$ is a small tolerance.

There are several ways to solve the MILP. One of the most efficient algorithms is a branch-and-bound method. Although it requires some heuristic rules in the decision of the branching variable, it can guarantee the optimality, and can reduce the computational burden with assistance of appropriate heuristic rules.

Note that the computational burden strongly depends on the number of binary variables since this specifies the 
size of the search space. In our case, the number of the measurement points affects the computational burden significantly.

\section{4 Identification results of stopping behavior based on PWPS model}

Based on the formulation of the identification of the PWPS model described in the previous section, the identification of parameters in the switching conditions and coefficients in the polynomials in the stopping behavior is carried out. The profile between the beginning of the deceleration and the final stopping point of each driver shown in Fig. 3 was used for the modeling.

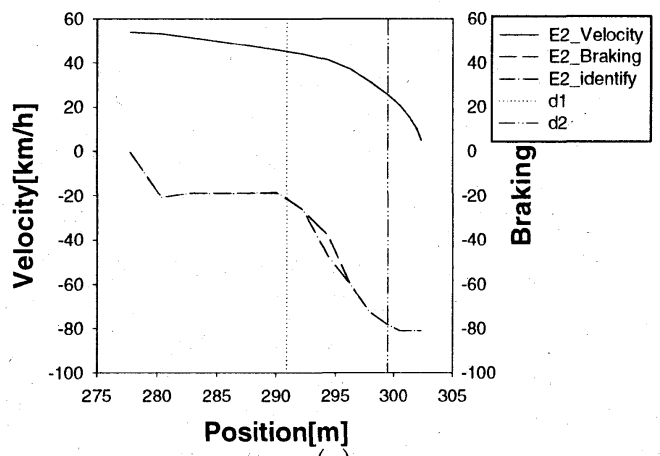

(a)

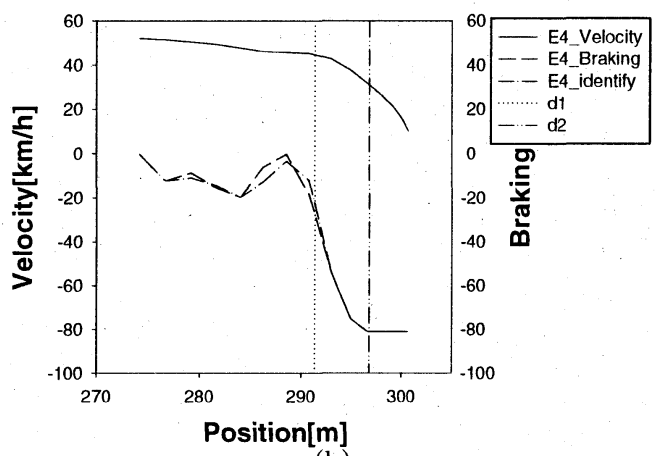

(b)

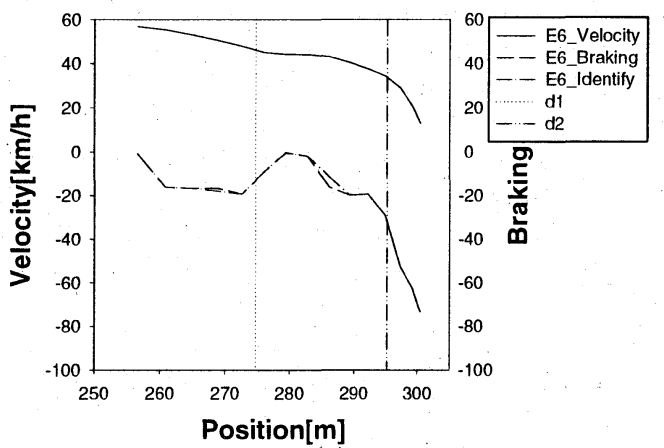

(c)

Fig. 4 Comparision of actual brake input and estimated brake input.

The fifteen sampling data of the third trial of six drivers were used for the identification of the stopping behavior. These data were selected by culling from the measured data between the beginning of the deceleration and the final stopping point. Before applying the MILP to the measured data, the input and output data were normalized as follows :

$$
\begin{gathered}
\bar{x}_{i}=\left\{\begin{array}{cc}
\frac{x_{i}}{x_{\text {imax. }}} & \left(x_{i} \geq 0\right) \\
-\frac{x_{i}}{x_{i m i n}} & \left(x_{i}<0\right)
\end{array}\right. \\
x_{i} \in\left[\begin{array}{ll}
x_{\text {imin }} & \left.x_{\text {imax }}\right]
\end{array}\right. \\
\bar{y}_{i}= \begin{cases}\frac{y_{i}}{y_{\text {iman. }}} & \left(y_{i} \geq 0\right) \\
-\frac{y_{i}}{y_{\text {imin }}} & \left(y_{i}<0\right)\end{cases}
\end{gathered}
$$$$
y_{i} \in\left[y_{\text {imin }} y_{\text {imax }}\right]
$$

All numerical experiments have been performed by $\mathrm{PC}$ (CPU $43.06[\mathrm{GHz}]$ and Memory 1024[MB]). It took about two hours to find the solution of the MILP. In order to verify the validity of the obtained PWPS model, the reproduced braking amount, which is calculated using the MILP, and the measured braking amount and the velocity of the vehicle are plotted in Fig. 4.

In Fig. 4, the horizontal axis represents the distance from the starting point. The left and right side vertical axes represent the velocity and braking amount of the vehicle, respectively. The stopping line is located at $300[\mathrm{~m}]$. Note that the braking amount takes negative values, and zero braking implies 'no braking'. Also, the switching points between polynomials are designated by vertical lines. As shown in Fig. 4, the measured braking amount and reproduced braking amount based on the PWPS model agree well with each other. These results verify the effectiveness of the modeling based on the PWPS. It is interesting that the switching points, however, vary from driver to driver. In order to understand the common characteristics in the stopping behavior, the identified parameters for three examinees (E2, E4 and E6)by solving the MILP are listed in Table 3.

Table 3 Identified parameters and values by a PWPS form of E2, E4 and E6

\begin{tabular}{c|c|c|c}
\hline \hline Parameters & Values(E2) & Values(E4) & Values(E6) \\
\hline$a_{0}$ & -0.4619 & -0.1501 & 0.9824 \\
\hline$a_{1}$ & $\underline{2.3118}$ & $\underline{1.1358}$ & $\underline{1.2932}$ \\
\hline$a_{2}$ & 0.5474 & 0.1834 & -1.1310 \\
\hline$\underline{a_{3}}$ & $\underline{4.4584}$ & $\underline{0.8705}$ & $\underline{1.8747}$ \\
\hline$b_{0}$ & $\underline{26.8100}$ & $\underline{24.2000}$ & $\underline{1.7534}$ \\
\hline$b_{1}$ & 1.6844 & -20.6600 & 0.4065 \\
\hline$b_{2}$ & -27.5780 & $\underline{-34.4040}$ & $\underline{-1.8845}$ \\
\hline$b_{3}$ & 1.2212 & -12.058 & -0.6546 \\
\hline$\underline{c_{0}}$ & -2.0241 & -2.0050 & $\underline{11.2200}$ \\
\hline$c_{1}$ & -0.0233 & 0.0039 & -20.6630 \\
\hline$c_{2}$ & $\underline{1.0130}$ & $\underline{1.0067}$ & -20.8540 \\
\hline$c_{3}$ & -0.0122 & 0.0023 & -10.029 \\
\hline$d_{1}$ & 290.900 & 291.360 & 274.739 \\
\hline$d_{2}$ & 299.477 & 296.776 & 295.222 \\
\hline \hline
\end{tabular}


The coefficients in the polynomials $a_{i}, b_{i}, c_{i}$ and the parameters in the switching conditions $d_{i}$ in(13) and (18) are listed in Table 3. As shown in Table 3, the most dominant input information (designated by underline in Table 3 ) in the intervals $\mathrm{B}$ and $\mathrm{C}$ were the distance $x_{1, k}$ to the stop line, and the acceleration $x_{3, k}$ did not play an important role in the stopping maneuver. On the other hand, the acceleration $x_{3, k}$ was found to be dominant input information, and played a crucial role in the interval A. Similar tendency has been found in the identified results of other drivers.

From these observation, we can conclude that the driver appropriately switches the 'control law' according to the following scenario: At the beginning of the stopping maneuver (just after finding the stopping point), the driver decelerate the vehicle based on the acceleration information, and then switch to another control law based on the distance to the stop line. Although the switching points depend on the driver's characteristics, qualitatively speaking, the scenario described above can be found as common characteristics in all drivers.

These results highly demonstrate the usefulness of the modeling based on the expression as an HDS.

\section{Conclusions}

A new framework to understand the human driving behavior based on the expression as HDS focusing on the driver's stopping maneuver has been developed. The driving data have been collected by using the threedimensional driving simulator based on CAVE, which provides three-dimensional visual information. In our modeling, the relationship between the measured information such as distance to the stop line, its first and second derivatives and the braking amount has been expressed by the PWPS model, which is a class of HDS. The key idea to solve the identification problem was to formulate the problem as the MILP with replacing the switching conditions by binary variables. From the obtained results, it was found that the driver appropriately switches the 'control law' according to the following scenario: At the beginning of the stopping behavior (just after finding the stopping point), the driver decelerates the vehicle based on the acceleration information, and then switches to the control law based on the distance to the stop line. Thus, our proposed approach enables us to capture not only the physical meaning of the driving skill, but also the decision-making aspect (switching conditions) in the driving behavior.

The analysis in more complicated situation, and the ap- plication of the obtained results to the design of Driving Assisting System are our future works.

\section{Acknowledgement}

This work was supported by the Space Robotic Center of the Toyota Technological Institute, where CAVE is installed. The authors would like to thank Dr. Yoji Umetani and the researchers involved at the Space Robotic Center for their helpful suggestions. The authors also would like to thank Dr. Yasushi Amano, of Toyota Central Research and Development Labs, for his valuable advices.

\section{References}

1) M.C. Nechyba and Y. Xu : Human Control Strategy: Abstraction, Verification and Replication, IEEE Control Systems Magazine, 17-5, 48/61 (1997)

2) Kim Jong-Hae, Yoshimitchi Matsui and Soichiro Hayakawa et al.: Acquisition and Modeling of Driving skill using Three-Dimensional Virtual System, Proceeding of ICASE/SICE Joint Workshop, 1, 49/53, October (2002)

3) Hiroshi Uno and Kaneo Hiramatsu: Aged Driver's Avoidance Capabilities in an Emergent Traffic situation, SAEJ, 32-1, 113/118, January (2001)

4) Alberto Bemporad, Giancarlo Ferrari-Trecate and Manfred Moarari: Observability and Controllability of Piecewise Affine and Hybrid Systems, IEEE Transaction on Automatic Control, 45-10, 1864/1876, October (2000)

5) Alberto Bemporad and Manfred Moarari: Control of Systems integration logic, dynamics, and constraints, Automatica, 35, 407/427, March (1999)

6) Alberto Bemporad, Jacob Roll and Lennart Ljung : Identification of Hybrid Systems via Mixed-Integer Programming, Proceedings of The 40th IEEE Conference on Decision and Control, 786/792, December (2001)

7) J.Sjoberg, Q..Zhang, L.Ljung, A.Benveniste, B.Deylon, P.Y. Glorenner, H.Hjalmarsson and A. Juditsky : Nonlinear Black-Box Modeling in System Identification: a Unified Overview, Automatica, 31-12, 1691/1724 (1995)

8) M.Akamatsu, S.Doi, M.Okuwa and K.Takuguchi : Behavior Based Human Environment Creation Technology, Project International Workshop on ITS Human Interface, Japan (ITS HMI symposium 2000)

9) S. Doi, Sueharu Nagiri et al.: Research and Development of Driving Support System for Individual Driving Ability, Toyota Central R and D Labs. Report, 71/107 (1998)

10) Aoi Kawashima, Kazuyaki Kobayashi et al. : Modeling on the Mental Stress and Automobile Driving, SICE, 38-1, 26/34 (2002)

11) Kiichi Yamada and Takashi Wakasugi : A Study on Effectiveness of Forward Vehicle Collision Warning, SAEJ, 32-1, 119/124 (2001)

12) Henry Been-Lirn Duh etc : An 'Independent Visual Background' Reduced Balance Disturbance Evoked by Visual Scene Motion: Implication for Alleviating Simulator Sickness, SIGCHI'01, Seattle, USA. (2001)

13) Maria T.Schultheis and Ronald R. Mourant: Virtual Reality and Driving: The Road to Better Assessment for Cognitively Impaired Populations : Presence by MIT, 10-4, 431/439, August (2001) 


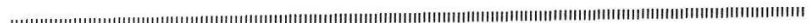

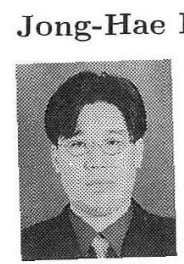

He was born in Ulsan, KOREA, in 1968. He received the M.E. and $\mathrm{PhD}$ degrees in Electrical Engineering from Yeungnam University, KOREA in 1996 and 1999, respectively. He is currently working toward the $\mathrm{PhD}$ degree at the Department of Electrical Engineering, Nagoya. University, JAPAN. His researh interests are in the area of resonant inverter, soft switching technology, virtual reality and hybrid dynamical systems.

\section{Soichiro Hayakawa (Member)}

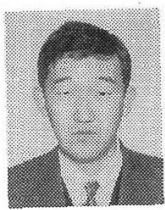

He was born in Aichi, JAPAN, in 1968. He received the $\mathrm{PhD}$ degree in Electrical Engineering from Nagoya University, JAPAN in 1996. Since 1996, he has been an Assistant Professor at Toyota Technological Institute, JAPAN. His researh interests are in the area of motion control systems, robotics, virtual reality and hybrid dynamical systems. Dr.Hayakawa is a member of the IEE.J and IEICE.

\section{Tatsuya Suzuki (Member)}

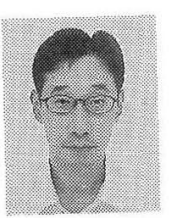

He was born in Aichi, Japan, in 1964. He received the B.E., M.E. and PhD degrees in Elcctronic Mechanical Engineering from Nagoya University, JAPAN in 1986, 1988 and 1991, respectively. From 1998 to 1999, he was a visiting researcher of the Mechanical Engineering Department of U.C.Berkeley. He is currently an Associate Professor of the Department of Electronic-Mechanical Engineering of Nagoya University. He won the paper award from IEEJ in 1995. His researh interests are in the area of motion control systems, hybrid dynamical systems, scheduling and so on. Dr.Suzuki is a member of the IEEJ, RSJ, JSME and IFEE.

\section{Kazuaki Hirana (Member)}

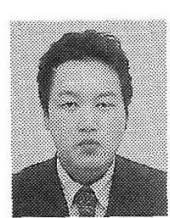

He was born in Aichi, JAPAN, in 1975. $\mathrm{He}$ received the B.E. and M.E. degrees in Electrical Engineering from Nagoya University, JAPAN in 1998 and 2000, respectively. Currently, he is an Assistant Professor at School of Computer and Cognitive Sciences, Chukyo University. His current research interests are in the areas of hybrid dynamical systems and motion control systems. Mr.Hirana is a member of the IEEJ and RSJ.
Yoshimichi MatsuI

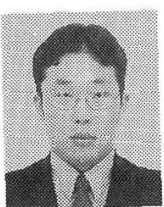

He was born in Aichi, JAPAN, in 1978. $\mathrm{He}$ received the B.E. and M.E. degrees in Electrical Engineering from Nagoya University, JAPAN in 2001 and 2003, respectively. He is currently working toward the $\mathrm{PhD}$ degree at the Department of Electronics and Information Engineering, Nagoya University, JAPAN. His researh interests include virtual reality and hybrid dynamical systems.

\section{Shigeru Okuma (Member)}

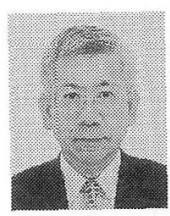

He was born in Gifu, JAPAN, in 1948. He received the B.E., M.E. and $\mathrm{PhD}$ degrees in Electronic-Mechnical Engineering from Nagoya University, JAPAN in 1970, 1972 and 1978, respectively. He also received the M.E. degree in systems engineering from Case Western Reserve University, Cleveland, OH, in 1974. Since 1990, he has been a Professor in the Department of Electronics and Information Engineering, Nagoya University. His researh interests are power elctronics, robotics and evolutionary soft computing. Dr. Okuma was the recipient of the IEEE IECON'92 Best Paper Award, in addition to paper awards from the Japan Society for Precision Engineering and Institute of Electrical Engineering of JAPAN.

\section{Nuio Tsuchida}

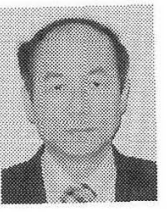

He was born in Aichi, JAPAN, in 1943. He received the $\mathrm{PhD}$ degree in Electrical and Electronics Engineering from Nagoya University, JAPAN in 1972.From 1972 to 1982 , he was an Assistant Professor in the Department of Electrical and Electronics Engineering, Nagoya University. Since 1992, he has been a Professor at Toyota Technological Institute. His researh interests are micro actuator, sensor and robotics. Dr. Tsuchida is a member of the IEEJ, IEICE and IEJ.

\section{Masayuki Sнimizu}

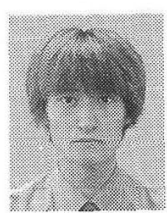

He was born in Chiba, JAPAN, in 1974. He received the B.E. degree in Electrical and Electronics Engineering and the M.E. degree in Electronics Machinery Science from Chiba University, JAPAN in 1997 and 1999, respectively. He entered Fujikura. Ltd in 1999. He developed Optical System Equipment and Optical Device(Fiber Bragg Grating). He entered Toyota Motor Corporation, Vehicle Control System Engineering Dept. in 2001. He has been working on Forward Collision Avoidance Assistance System. 
Shigeyuki KIDo

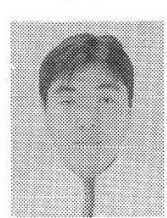

He was born in Tokyo, JAPAN, in 1963. He received the B.E.and M.E. degrees in Machanical Sience from Nagoya University, JAPAN in 1986 and 1988, respectively. He entered Toyota Motor Corporation in 1988. Since 1988, he has been working on Vehicle Control System. 